\title{
Synthesis of Some New Bisindole Derivatives and Their Biological Activity
}

\author{
Amal S. Yanni \\ Chemistry Department, Faculty of Science, Assiut University, Assiut, Egypt \\ Email: Profayanni@yahoo.com
}

How to cite this paper: Yanni, A.S. (2016) Synthesis of Some New Bisindole Derivatives and Their Biological Activity. International Journal of Organic Chemistry, 6, 187191.

http://dx.doi.org/10.4236/ijoc.2016.64019

Received: March 7, 2016

Accepted: November 8, 2016

Published: November 11, 2016

Copyright $\odot 2016$ by author and Scientific Research Publishing Inc. This work is licensed under the Creative Commons Attribution International License (CC BY 4.0). http://creativecommons.org/licenses/by/4.0/

\begin{abstract}
Substituted 1,4-diaza-1,3-butadienes with 2 equivalents of 1,4-naphthoquinone in presence of ethanol or benzene produce corresponding substituted bisindoletetrones via criss-cross cycloaddition reaction. The chemical structure was confirmed by elemental and spectral analysis. Biological activity against some micro-organisms was tested.
\end{abstract}

\section{Keywords}

Synthesis, Bisindoletetrones, Cycloaddition, Antimicrobial Activity

\section{Introduction}

Pyrrolidines are well known for their versatile pharmacological activities such as antimicrobial [1] [2] [3] [4], antitumor [5], anti HIV [6], anticonvulsant [7] [8], human melanocortin-4 receptor agonists [9], etc. Moreover, indole nucleus is an important element of many natural and synthetic molecules that covers some of the relevant and recent achievement in the biological, chemical and pharmaceutical activity of important indole derivatives [10]. In view of these observations, the intention is directed to synthesize some new bisindole derivatives of expected biological interest.

\section{Experimental}

\subsection{General}

All melting points are uncorrected. IR spectra were recorded $(\mathrm{KBr})$ with a Perkin- Elmer 1430 spectrophotometer. ${ }^{1} \mathrm{H}$ NMR spectra were obtained on Varian EM 399.65 $\mathrm{MHz}$ equipment. MS spectra were recorded with a Jeol the MS route JMS-600 H. Substituted 1,4-diaza-1,3-butadienes (1a-f) were prepared according to known procedures by condensation of glyoxal and aromatic amines. 


\subsection{6,13-Disubstituted 6,6a,13,13a-Tetrahydro-(5aH,5bH,12aH, 12bH)-Bisbenzo[f]Benzo-[5,6]Indolo[3,2-b]Indole-5,7,12,14- Tetrone (3a-f): General Procedure}

A mixture of substituted 1,4-diaza-1,3-butadienes $(1,1 \mathrm{mmole})$ and 1,4-naphthoquinone (2, 2 mmoles) was refluxed in absolute ethanol or benzene for $8-12 \mathrm{hrs}$. The solution was filtered while hot. The mother liquor was concentrated and cooled. The highly colored (brown violet) products were filtered off and crystallized from ethanol. Yield $40 \%$ $-55 \%$.

\subsubsection{6,13-Diphenyl-6,6a,13,13a-Tetrahydro-(5aH,5bH,12aH,} 12bH)-Bisbenzo[f]Benzo-[5,6]Indolo[3,2-b]Indole-5,7,12,14-Tetrone (3a)

Adduct (3a), $\mathrm{R}=-\mathrm{C}_{6} \mathrm{H}_{5}$, is obtained from 1,4-diphenyl-1,4-diazabuta-1,3-diene (1a) and (2) in 50\% yield, m.p. $184^{\circ} \mathrm{C}$. Anal. calcd. for $\mathrm{C}_{34} \mathrm{H}_{24} \mathrm{~N}_{2} \mathrm{O}_{4}$ (524.58): C, 77.85; H, 4.61; N, 5.34. Found: C, 77.58; H, 4.79; N, 5.42. IR $\left(\mathrm{cm}^{-1}\right)$, v: $1669(\mathrm{C}=\mathrm{O}), 1260,1445$ (C-N). MS m/z (\%): [ $\left.\mathrm{M}^{+}-\mathrm{PhCO}-\mathrm{O}-\mathrm{H}\right] 402(0.1)$, common fragment ion $\left[\mathrm{M}^{+}-2 \mathrm{C}_{6} \mathrm{H}_{5}\right.$ 2O] $337.8(10.7)$.

\subsubsection{6,13-Di-p-Tolyl-6,6a,13,13a-Tetrahydro-(5aH,5bH,12aH, 12bH)-} Bisbenzo[f]Benzo-[5,6]Indolo[3,2-b]Indole-5,7,12,14-Tetrone (3b) Adduct (3b), $\mathrm{R}=-\mathrm{C}_{6} \mathrm{H}_{4}-p$ - $\mathrm{CH}_{3}$, is obtained from 1,4-di- $p$-tolyl-1,4-diaza-1,3-butadiene (1b) and (2) in $40 \%$ yield, m.p. $188^{\circ} \mathrm{C}-190^{\circ} \mathrm{C}$. Anal. calcd. for $\mathrm{C}_{36} \mathrm{H}_{28} \mathrm{~N}_{2} \mathrm{O}_{4}$ (552.64): C, 78.24; H, 5.11; N, 5.07. Found: C, 78.37; H, 5.26; N, 5.14. IR $\left(\mathrm{cm}^{-1}\right), v: 1670(\mathrm{C}=\mathrm{O})$, 1246, $1508(\mathrm{C}-\mathrm{N}) .{ }^{1} \mathrm{H}$ NMR $\left(\mathrm{CDCl}_{3}\right), \delta: 2.44\left(\mathrm{~s}, 6 \mathrm{H}, 2-\mathrm{CH}_{3}\right), 3.7(2 \mathrm{H}, 2 \mathrm{CH}-\mathrm{N}), 6.3(2 \mathrm{H}$, 2-CH-CO), $6.8-8.2(\mathrm{~m}, 2 \mathrm{H}, 2-\mathrm{CO}-\mathrm{CH}-\mathrm{N}-, 16 \mathrm{H}, \mathrm{Ar}-\mathrm{H}) . \mathrm{MS} \mathrm{m} / \mathrm{z}(\%):\left[\mathrm{M}^{+}-2 \mathrm{O}-\mathrm{C}_{2}\right]$ 466.6 (0.2), common fragment ion $\left.] \mathrm{M}^{+}-2 \mathrm{C}_{6} \mathrm{H}_{4}-p-\mathrm{CH}_{3}-2 \mathrm{O}\right] 337.79$ (11.4).

\subsubsection{6,13-Di-p-Methoxyphenyl-6,6a,13,13a-Tetrahydro-(5aH,5bH,} 12aH,12bH)-Bisbenzo-[f]Benzo[5,6]Indolo[3,2-b]Indole-5,7,12,14Tetrone (3c)

Adduct (3c), $\mathrm{R}=-\mathrm{C}_{6} \mathrm{H}_{4}-p-\mathrm{OCH}_{3}$, is obtained from 1,4-di- $p$-methoxyphenyl-1,4-diaza1,3-butadiene (1c) and (2) in $42 \%$ yield, m.p. $140^{\circ} \mathrm{C}$. Anal. calcd. for $\mathrm{C}_{36} \mathrm{H}_{28} \mathrm{~N}_{2} \mathrm{O}_{6}$ (584.63): C, 73.96; H, 4.83; N, 4.79. Found: C, 73.49; H, 4.68; N, 4.90. IR $\left(\mathrm{cm}^{-1}\right), v: 1670$ $(\mathrm{C}=\mathrm{O}), 1249,1508(\mathrm{C}-\mathrm{N}) . \mathrm{MS} \mathrm{m} / \mathrm{z}(\%):\left[\mathrm{M}^{+}-\mathrm{Ph}-\mathrm{C}_{2}\right] 483.45$ (0.2), common fragment ion $\left[\mathrm{M}^{+}-2 \mathrm{C}_{6} \mathrm{H}_{4}-p-\mathrm{OCH}_{3}-2 \mathrm{O}\right] 337.9$ (0.9).

\subsubsection{6,13-Di-p-Hydroxyphenyl-6,6a,13,13a-Tetrahydro-(5aH,5bH,} 12aH,12bH)-Bisbenzo-[f]Benzo[5,6]Indolo[3,2-b]Indole-5,7,12,14Tetrone (3d)

Adduct (3d), $\mathrm{R}=-\mathrm{C}_{6} \mathrm{H}_{4}-p$-OH, is obtained from 1,4-di- $p$-hydroxyphenyl-1,4-diaza-1,3butadiene (1d) and (2) in $45 \%$ yield, m.p. $240^{\circ} \mathrm{C}$. Anal. calcd. for $\mathrm{C}_{34} \mathrm{H}_{24} \mathrm{~N}_{2} \mathrm{O}_{6}(556.58)$ : C, 73.37; H, 4.35; N, 5.03. Found: C, 73.19; H, 4.22; N, 4.90. IR $\left(\mathrm{cm}^{-1}\right), v: 3300(\mathrm{OH})$, $1671(\mathrm{C}=\mathrm{O}), 1267,1435(\mathrm{C}-\mathrm{N}) .{ }^{1} \mathrm{H}$ NMR $\left(\mathrm{CDCl}_{3}\right): \delta 3.7(\mathrm{~s}, 2 \mathrm{H}, \mathrm{N}-\mathrm{CH}-\mathrm{CH}-\mathrm{N}), 6.2(\mathrm{~s}$, $2 \mathrm{H}, 2-\mathrm{CH}-\mathrm{CO}$ ), 6.88 - 8.2 (m, 2H, 2-CO-CH-N, 16H, Ar-H), 8.6 (s, 2H, 2-OH). MS m/z (\%): $\left[\mathrm{M}^{+}-2 \mathrm{C}_{6} \mathrm{H}_{4}-\mathrm{OH}-2 \mathrm{O}\right] 338.2$ (3.8), common fragment ion $\left[\mathrm{M}^{+}-2 \mathrm{C}_{6} \mathrm{H}_{4} \mathrm{OH}-2 \mathrm{O}\right]$ $337.92(6.5)$. 


\subsubsection{6,13-Di-p-Chlorophenyl-6,6a,13,13a-Tetrahydro-(5aH,5bH, 12aH,12bH)-Bisbenzo-[f]Benzo[5,6]Indolo[3,2-b]Indole-5,7,12,14- Tetrone (3e)}

Adduct (3e), $\mathrm{R}=-\mathrm{C}_{6} \mathrm{H}_{4}-p$-Cl, is obtained from 1,4-di- $p$-chlorophenyl-1,4-diaza-1,3butadiene (1e) and (2) in 50\% yield, m.p. $205^{\circ} \mathrm{C}$. Anal. calcd. for $\mathrm{C}_{14} \mathrm{H}_{10} \mathrm{~N}_{2} \mathrm{Cl}$ (593.47): C, 68.81; H, 3.74; Cl, 11.95; N, 4.72. Found: C, 68.69; H, 3.61; Cl, 11.68; N, 4.91. IR $\left(\mathrm{cm}^{-1}\right), v: 1670(\mathrm{C}=\mathrm{O}), 1299,1406(\mathrm{C}-\mathrm{N}) . \mathrm{MS} \mathrm{m} / \mathrm{z}(\%):\left[\mathrm{M}^{+}-\mathrm{PhCO}-\mathrm{H}\right] 487$ (1.3), common fragment ion $\left[\mathrm{M}^{+}-\mathrm{C}_{6} \mathrm{H}_{4}-\mathrm{p}-\mathrm{Cl}-2 \mathrm{O}\right] 337.9$ (100).

\subsubsection{6,13-Di- $\alpha$-Naphthyl-6,6a,13,13a-Tetrahydro-(5aH,5bH,12aH, 12bH)- Bisbenzo-[f]Benzo[5,6]Indolo[3,2-b]Indole-5,7,12,14-Tetrone (3f)}

Adduct (3f), $\mathrm{R}=-\mathrm{C}_{10} \mathrm{H}_{7}$, is obtained from 1,4-di- $\alpha$-naphthyl-1,4-diaza-1,3-butadiene (1f) and (2) in $38 \%$ yield, m.p. $215^{\circ} \mathrm{C}$. Anal. calcd. for $\mathrm{C}_{42} \mathrm{H}_{28} \mathrm{~N}_{2} \mathrm{O}_{4}$ (624.7): C, 80.75; H, 4.52; N, 4.48. Found: $\mathrm{C}, 80.29 ; \mathrm{H}, 4.79 ; \mathrm{N}, 4.61$. IR $\left(\mathrm{cm}^{-1}\right), v: 1670(\mathrm{C}=\mathrm{O}), 1269,1455$ (C-N). MS m/z (\%): $\left[\mathrm{M}^{+}-2 \mathrm{C}_{10} \mathrm{H}_{7}-\mathrm{C}_{2} \mathrm{H}_{2}-2 \mathrm{H}\right] 342(0.1)$, common fragment ion $\left[\mathrm{M}^{+}-2\right.$ $\left.\mathrm{C}_{10} \mathrm{H}_{7}-2 \mathrm{O}\right] 337.9(20.9)$.

\subsection{Antimicrobial Activity*}

Antibacterial screening of compounds (3a-f) were determined by Nutrient agar well diffusion method. The tested compounds (3a-f) were dissolved in ethylene glycol to give $2 \%$ concentration. Antibacterial activity was determined according to the method reported by Bauer et al. [11] using $3 \mathrm{~mm}$ filter paper discs (Watmann No. 2) loaded with $10 \mu \mathrm{L}$ of the solution under investigation $(2.0 \%)$. The discs were placed on the surface of the bacterial culture, which were incubated at $30^{\circ} \mathrm{C}$. The diameter of the inhibition zone around each disc was measured (cf. Table 1).

\section{Results and Discussion}

Wagner [12] found that heating one mole of benzalazine with 2 moles of maleic anhydride to $100^{\circ} \mathrm{C}$ in dry benzene for several hours gave addition compound through simultaneous 1,3- \& 2,4-additoin of 2 moles of maleic anhydride to benzalazine, which was designated as criss-cross addition [13]. Moreover, aromatic 1,4-disubstituted 1,4-diazabuta-1,3-dienes with thiocyanates in glacial acetic acid via criss-cross cycloaddition produced the corresponding perhydroimidazo[4,5-d]imidazole-2,5-dithiones [14]. Similarly as continuation of my previous work [15] [16] [17] [18] [19], for the

Table 1. Bactericidal activity expressed as inhibition zone in $\mathrm{mm}$.

\begin{tabular}{ccccc}
\hline Organism & $\begin{array}{c}\text { Rhodopseudomonas } \\
\text { Compd. }\end{array}$ & $\begin{array}{c}\text { Bacillus } \\
\text { cereus }\end{array}$ & Micrococus luteus & $\begin{array}{c}\text { E. coli } \\
\text { (HD701) }\end{array}$ \\
\hline (3a) & 5 & - & 5 & 13 \\
$(3 b)$ & 12 & - & 12 & - \\
$(3 c)$ & 15 & - & - & 19 \\
$(3 d)$ & 7 & 5 & 17 & 25 \\
$(3 e)$ & 6 & 17 & - & - \\
(3f) & - & - & - & - \\
\hline \hline
\end{tabular}

${ }^{\star}$ Done by Dr. Amal W. Danial, Botany \& Microbiology Department, Faculty of science, Assiut University, Assiut 71526, Egypt. 


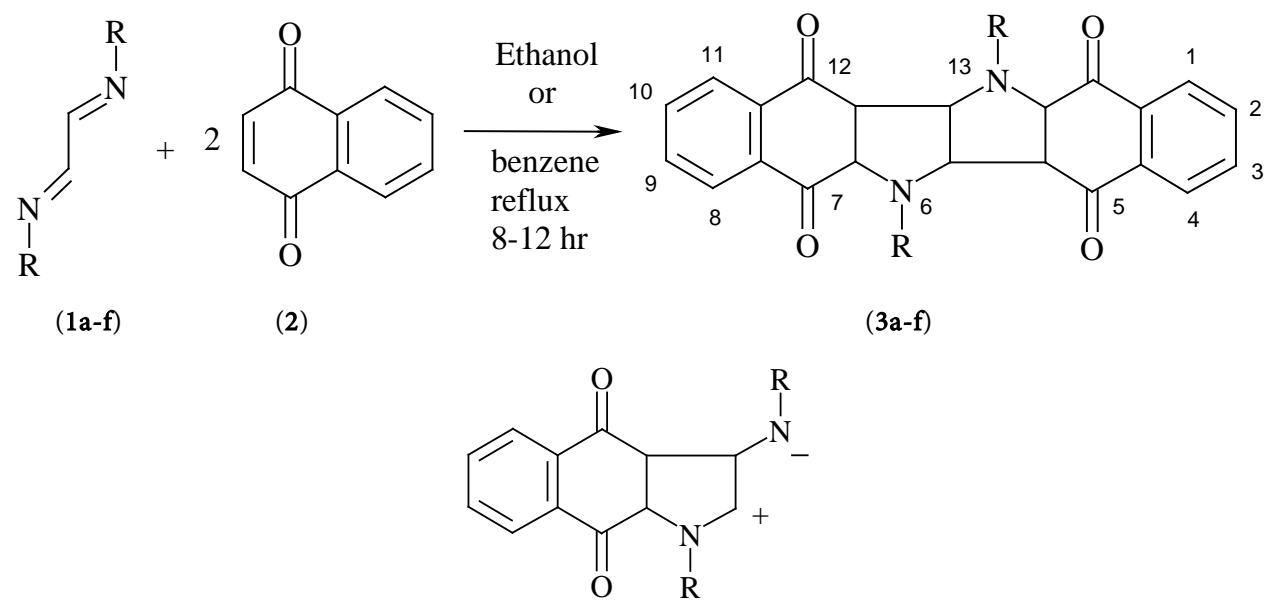

(4)

Scheme. $\mathrm{R}=-\mathrm{C}_{6} \mathrm{H}_{5}(1 \mathrm{a}, 3 \mathrm{a}) ;-\mathrm{C}_{6} \mathrm{H}_{4}-p-\mathrm{CH}_{3}(1 \mathrm{~b}, 3 \mathrm{~b}) ;-\mathrm{C}_{6} \mathrm{H}_{4}-p-\mathrm{OCH}_{3}(1 \mathrm{c}, 3 \mathrm{c}) ;-\mathrm{C}_{6} \mathrm{H}_{4}-p-\mathrm{OH}(1 \mathrm{~d}$, 3d); $-\mathrm{C}_{6} \mathrm{H}_{4}-p-\mathrm{Cl}(1 \mathbf{e}, 3 \mathbf{e}) ;-\mathrm{C}_{10} \mathrm{H}_{7}(\mathbf{1 f}, 3 \mathbf{f})$

following criss-cross cycoaddition (Scheme), substituted 1,4-diaza-1,3-butadienes (1af) were allowed to react with 2 equivalents of 1,4-naphthoquinone (2), one pot reaction in presence of ethanol or benzene and afforded bisindoletetrone derivatives (3a-f). The structure of the synthesized compounds was assigned by elemental and spectral analysis.

Huisgen [20] suggested that criss-cross reaction can be represented by series of two $[3+2]$ cycloaddition steps.

Compound (4) was postulated as a key intermediate. The compounds produced satisfactory results for elemental and spectral analysis.

As for their antimicrobial properties, some compounds exhibited strong activity such as (3c) (against Rhodopseduomonas fp. \& E. coli [HD701]), (3d) (against Micrococcus luteus \& E. coli), and (3e) (against Bacillus cereus). Others showed remarkable activity such as (3a) (against E. coli [HD701]), (3b) (against Micrococcus luteus \& Rhodopseudomonas $f p$.) while other compounds showed week or no potency (3f).

\section{Conclusion}

The title compounds are synthesized successfully through simple novel route and one pot reaction from substituted 1,4-diaza-1,3-butadienes and 1,4-naphthoquinone. These compounds also exhibited strongly to remarkable bactericidal activity against some tested bacteria.

\section{References}

[1] Babu, M.N., et al. (2012) Synthesis and Antimicrobial Activity of Some Novel Pyrrolidine Derivatives. International Journal of Chem Tech Research, 4, 903-909.

[2] Lokhande, T.N., Bobade, A.S. and Khadse, B.G. (2003) Synthesis of 4-(Pyrrolidin Anilino)$\mathrm{N}$-Aryl/Substituted Aryl and Heteroaryl Succinimides as Animicrobial and Antifungal Agents. Indian Drugs, 40, 147-150. 
[3] Mary, E.H., Gregory, B., Victor, N. and Adel, N. (2006) Pyrrolidine Bis-Cyclic Guanidines with Antimicrobial Activity against Drug-Resistant Gram-Positive Pathogenes Identified from a Mixture-Based Combinatorial Library. Bioorganic \& Medicinal Chemistry Letters, 16, 5073-5079. http://dx.doi.org/10.1016/j.bmcl.2006.07.037

[4] Donas, H.A., Yaha, N., Nizami, D. and Colin, K. (2006) Synthesis, Crystal Struture and Antifungal/Antibacterial Activity of Some Novel Highly Functionalized Benzoylaminocarbothioyl 1-Pyrrolidines. Turkish Journal of Chemistry, 30, 573-583.

[5] Li, X., Li, Y.L. and Xu, W.F. (2006) Design, Synthesis and Evaluation of Novel Galloyl Pyrrolidine Derivatives as Potential Antitumor Agents. Bioorganic \& Medicinal Chemistry, 14, 1287-1293. http://dx.doi.org/10.1016/j.bmc.2005.09.031

[6] Shinichi, I., Yugi, I., Taeko, H., Osamu, K., et al. (2004) Synthesis and Biological Evaluation of 5-Oxopyrrolidine-3-carboxamide Derivatives. Chemical and Pharmaceutical Bulletin, 52, 63-73. http://dx.doi.org/10.1248/cpb.52.63

[7] Jolanta, O. and Agniesk, Z. (2002) Synthesis and Anticonvulsant Properties of New N-(4Arylpiperazin-1-yl)-Methyl Derivatives of 3-Arylpyrrolidine-2,5-Dione and 2-Aza- Spiro [4,4] Nonane-1,3-Dione. IL Farmaco, 58, 1227-1234.

[8] Barbara, M. (2005) New Anticonvulsant Agents. Current Topics in Medicinal Chemistry, 5, 69-85. http://dx.doi.org/10.2174/1568026053386944

[9] Joe, A.T., Chen Caroline, W., et al. (2007) Pyrrolidines as Potent Functional Agonists of the Human Melanocortin-4 Receptor. Bioorganic \& Medicinal Chemistry Letters, 17, 51655170. http://dx.doi.org/10.1016/j.bmcl.2007.06.088

[10] Kaushik, N.K., et al. (2013) Biomedical Importance of Indoles. Molecules, 18, 6620-6662. http://dx.doi.org/10.3390/molecules18066620

[11] Baurer, A.W., Kibry, W.M., Sherris, J.C. and Truck, M. (1966) Antibiotic Susceptibility Testing by a Standardized Single Disk Method. American Journal of Clinical Pathology, 45, 493-496.

[12] Wagner-Jauregg, T. (1930) Uber Addierende Hetero-Polymerisation. Chemische Berichte, 63, 3218-3224. http://dx.doi.org/10.1002/cber.19300631140

[13] Van Alphen, J. (1942) The Reaction of Azines with Maleic Acid Anhydride. Recueil des Travaux Chimiques des Pays-Bas, 61, 892-895. http://dx.doi.org/10.1002/recl.19420611211

[14] Verner, J. and Potacek, M. (2004) Aromatic Glyoxalimines in Criss-Cross Cycloaddition. Central European Journal of Chemistry, 2, 220-233. http://dx.doi.org/10.2478/bf02476192

[15] Yanni, A.S. (2002) Synthesis of Some New Bispyrrolidine Derivatives for Biological Interest. 16th International Conference on Physical Organic Chemistry, San Diego, 4-9 August 2002.

[16] Yanni, A.S. (2004) Synthesis of Some New Bispyrrolidine Derivatives for Biological Interest: Part II. Polymer Networks Conference, Bethesda, 15-19 August 2004.

[17] Yanni, A.S. (2006) Synthesis of Some New Bipyrrolidine Derivaties for Biological Interest: Part III. 10th Annual Green Chemistry Conference, Washington DC, 24-29 June 2006.

[18] Yanni, A.S. (2008) Synthesis of Some New Bispyrrolidine Derivatives for Biological Interest: Part IV. 12th Annual Green Chemistry Conference, Washington DC, 23-27 June 2008.

[19] Yanni, A.S. (2014) Synthesis of Some New Bistriazolidine Derivatives and Their Biological Activity. Russian Journal of Bioorganic Chemistry, 40, 377-378. http://dx.doi.org/10.1134/S1068162014020150

[20] Huisgen, R. (1963) 1,3-Dipolar Cycloadditions: Past and Future. Angewandte Chemie, 2, 565-598. http://dx.doi.org/10.1002/anie.196305651 
Submit or recommend next manuscript to SCIRP and we will provide best service for you:

Accepting pre-submission inquiries through Email, Facebook, LinkedIn, Twitter, etc. A wide selection of journals (inclusive of 9 subjects, more than 200 journals)

Providing 24-hour high-quality service

User-friendly online submission system

Fair and swift peer-review system

Efficient typesetting and proofreading procedure

Display of the result of downloads and visits, as well as the number of cited articles

Maximum dissemination of your research work

Submit your manuscript at: http://papersubmission.scirp.org/

Or contact ijoc@scirp.org 